\title{
CIRCOVIRUS PORCINO: UN VIRUS PEQUEÑO QUE GENERA UN GRAN PROBLEMA
}

\author{
PORCINE CIRCOVIRUS: A SMALL VIRUS THAT CAUSES A GREAT PROBLEM
}

\author{
Jorge NoriegA ${ }^{1}$, PAulina Reyes ${ }^{1}$, Sergio BuCAReY ${ }^{1}$
}

\begin{abstract}
Porcine circoviruses type 1 and type 2 (PCV-1 and PCV-2) are very small (17 nm), nonenveloped, icosahedral viruses (Tisher y col., 1982), with circular, single stranded DNA genomes of approximately 1,700 nucleotides length, encoding 2 major ambisense open reading frames (Mankertz y col., 1998; Hamel y col., 1998). Together with a number of avian viruses with similar molecular characteristics, the porcine circoviruses are classified in the genus Circovirus within the family Circoviridae. Although $P C V-1$ persists in the pig population, the presence of $P C V-1$ has not been associated with any recognised clinical signs or pathology. In contrast, PCV-2 has been implicated as the mayor causative agent of Postweaning Multisystemic Wasting Syndrome (PMWS). It is characterized mainly by loose of weight, general wasting, and severe immunosuppressive effects with premature death of piglets.

$P C V-2$ effects are generated by inhibition of plasmacytoid dendritic cells ( $p D C$ ) so called natural interferon- $\alpha$ producing cells (NIPCs) unlinking the innate and acquired immune systems, and rendering a host susceptible to secondary or concomitant microbial infections. Therefore, $P C V-2$ additionally is associated with other porcine diseases and syndromes, such as reproductive failure, respiratory disease complex, congenital tremor, porcine dermatitis and nephropathy syndrome, nectrotizing tracheitis and exudative epidermitis, together with other viral and bacterial agents, including Parvovirus, PRRSV (Porcine Reproductive and Respiratory Syndrome virus), Influenza, Aujeszky and Mycoplasma pneumoniae, Actinobacillus pleuropneumoniae, Streptococcus suis and Pastereulla multocida. Nowadays, these diseases are known as PCVAD or PCV-2-associated diseases, in order to group all the diseases attributed to porcine circovirus type 2.

The PCV-2-associated diseases, mainly PMWS, at present are considered to be the greatest economic problem associated with the pig industry world-wide.
\end{abstract}

KEY WORDS: $P C V$-2, circovirus, wasting diseases, PCVAD.

\section{RESUMEN}

\begin{abstract}
Los circovirus porcinos tipo 1 y tipo 2 (PCV-1 y PCV-2) son virus muy pequeños (17 nm), no envueltos, de simetría icosahédrica (Tisher y col., 1982), con un DNA circular de hebra simple de aproximadamente 1.700 nucleótidos de longitud, el cual codifica principalmente dos marcos de lectura abiertos (Mankertz y col., 1998; Hamel y col., 1998). En conjunto con un número de virus aviares con características moleculares similares, los circovirus porcinos se clasifican en el género Circovirus dentro de la familia Circoviridae. Aunque el PCV-1 es persistente en la población de cerdos, la presencia de PCV-1 no ha sido asociada con ninguna signología clínica o patología reconocida. En contraste, PCV-2 ha sido implicado como el agente etiológico principal del Síndrome Multisistémico de Desmedro Postdestete (PMWS), caracterizado principalmente por pérdida de peso, deterioro general de la condición corpórea y efectos inmunosupresivos severos, los que conllevan a la muerte prematura de los lechones.
\end{abstract}

Centro Biotecnológico Veterinario Biovetec y Departamento de Ciencias Biológicas Animales, Facultad de Ciencias Veterinarias y Pecuarias, Universidad de Chile. 
Los efectos de PCV-2 son generados por inhibición de las células dendríticas plasmocitoideas ( $p D C)$, también llamadas células productoras naturales de interferon- $\alpha$ (NIPCs), afectando al sistema inmune innato $y$ adquirido, $y$ dejando al hospedero susceptible a infecciones microbianas secundarias o concomitantes. De esta manera, PCV-2 adicionalmente se asocia a otras enfermedades y síndromes porcinos, como la falla reproductiva, enfermedad del complejo respiratorio, tremor congénito, síndrome de dermatitis y nefropatia porcina, traqueítis necrotizante y epidermitis exudativa, en conjunto con otros agentes virales y bacterianos, incluyendo al Parvovirus, PRRSV (virus del sindrome reproductivo y respiratorio porcino), Influenza, Aujeszky y Mycoplasma pneumoniae, Actinobacillus pleuropneumoniae, Streptococcus suis and Pasteurella multocida. Hoy en dia estas enfermedades son conocidas como PCVAD o enfermedades asociadas a circovirus porcino, de forma de agrupar a todas las enfermedades atribuidas al circovirus porcino tipo 2, incluida el PMWS.

Las PCVAD son consideradas, actualmente, como el mayor problema económico asociado a la industria porcina mundial.

Palabras ClaVe: PCV-2, circovirus, enfermedad del desmedro, PCVAD.

\section{Historia del circovirus porcino}

$\mathrm{El}$ circovirus porcino ( $\mathrm{PCV}$, del inglés porcine circovirus) fue descrito por primera vez por investigadores alemanes en el año 1974, como un virus contaminante de la línea celular de riñón de cerdo PK-15 (ATCC-CCL33), el cual fue denominado inicialmente como picornavirus-like (Tischer $y$ col., 1974). Estudios posteriores revelaron que se trataba de un virus muy pequeño, sin envoltura, con un diámetro de $17 \mathrm{~nm}$ y de simetría icosaédrica, el cual presentaba un genoma de DNA circular, por lo que fue denominado circovirus porcino (PCV) (Tischer $y$ col., 1982).

En el año 1997 el PCV fue asociado a una enfermedad que afectaba a cerdos de transición conocida como Síndrome Multisistémico de Desmedro Postdestete (PMWS, del inglés Postweaning Multysistemic Wasting Syndrome) (Clark, 1997). Esta enfermedad ya era conocida desde 1991 en Canadá, pero se consideraba de etiología desconocida (Harding, 1996; Clark 1997; Harding y clark 1997; Harding y col., 1998). Los síntomas observados eran pérdida de peso, palidez corporal, alteraciones respiratorias y, en algunos casos, diarrea e ictericia. Las lesiones más frecuentemente observadas eran neumonía intersticial y linfoadenopatía generalizada, especialmente en los nódulos linfáticos inguinales superficiales. Otras lesiones que se observan con menor frecuencia eran hepatitis, nefritis y pancreatitis no supurativas (Harding, 1996; Clark 1997; Harding y clark 1997; Harding y col., 1998; Rosell y col., 1999). En los tejidos lesionados se observaba sistemáticamente una gran cantidad de antígeno y ácido nucleico viral (Clark, 1997; Rosell y col., 1999).
Estudios posteriores de secuenciación genómica demostraron que el genotipo de PCV presente en cerdos afectados con PMWS era diferente al genotipo de PCV que contaminaba persistentemente la línea celular PK-15 (Hamel y col., 1998; Meehan y col., 1998). Por ello se sugirió la denominación de PCV tipo 1 (PCV-1) para el circovirus asociado a la línea celular PK-15, considerado apatógeno, y PCV tipo 2 (PCV-2) para el circovirus asociado a PMWS (Allan y col., 1998; Meehan y col., 1998). De esta forma, hasta la fecha se ha utilizado la nomenclatura PCV-1 y PCV-2 para designar a los dos genotipos virales.

\section{La familia Circoviridae}

La familia Circoviridae está dividida en dos géneros, Gyrovirus y Circovirus. El género Circovirus agrupa a: PCV-1, PCV-2, virus de la enfermedad del pico y de las plumas de las psitácidas (BFDV, del inglés Beak and Feather Disease Virus) (Bassami y col., 1998; Niegro y col., 1998), circovirus de la paloma (PiCV, del inglés Pigeon Circovirus o $\mathrm{CoCV}$, del inglés Columbid Circovirus) (Mankertz $y$ col., 2000), circovirus del ganso (GCV, del inglés Goose Circovirus) (Todd y col., 2001), circovirus del canario (CaCV, del inglés Canary Circovirus) (Phenix y col., 2001) y circovirus de gaviotas (GuCV, del inglés Gull Circovirus) (Jestin y col., 2001). Al género Gyrovirus pertenece el virus de la anemia del pollo (CAV, del inglés Chiken Anemia Virus) (Todd y col., 1990).

\section{Características fisicoquímicas de los PCVs}

Los circovirus porcinos presentan un peso molecular de $36 \pm 2,3 \mathrm{kDa}$, una densidad de sedimentación en 
$\mathrm{CsCl}$ entre 1,33 y $1,37 \mathrm{~g} / \mathrm{cm}^{3}$ y un coeficiente de sedimentación de $52 \mathrm{~S}$ (Tisher y col., 1974). Según datos referidos a PCV-1 indican que se trata de un virus resistente a la inactivación por cloroformo, al $\mathrm{pH}$ ácido y termoestable a $70{ }^{\circ} \mathrm{C}$ durante 15 minutos (Allan y col., 1994).

\section{Genoma de PCV-2}

Estudios de secuenciación indican que el genoma del PCV-2 contiene sólo 1.767-1.768 nucleótidos de largo (Hamel y col., 1998; Meehan y col., 1998; Mankertz y col., 2000). Además, se ha descrito más de un $94 \%$ de identidad nucleotídica entre los PCV-2, aislados tanto desde cerdos enfermos como sanos, alrededor del mundo (Mehhan y col., 1998; Mankertz y col., 2000; Larochelle y col., 2002). Sin embargo, al comparar las secuencias genómicas de aislados de PCV-2 con respecto a PCV-1, se muestra sólo un $80 \%$ de identidad nucleotídica. El genoma de los circovirus se caracteriza por tener una región que codifica proteínas o marcos de lectura abiertos (ORFs, del inglés Open Reading Frames) y una región no codificante involucrada en regular la transcripción y replicación del virus (Hamel y col., 1998). Se han descrito potencialmente 6 ORFs con más de 200 nucleótidos para el genoma de PCV-2 (Hamel y col., 1998; Meehan y col., 1998), pero las proteínas esenciales para la replicación y para la conformación estructural del virus son codificadas principalmente por ORF1 y ORF2 respectivamente, siendo estos fragmentos los más estudiados y caracterizados (Segalés $y$ col., 2005). La proteína de la cápside viral (Cap) es codificada tras un proceso de "splicing" por la hebra complementaria del ORF2 (Cheung, 2002). Como es de esperar en una proteína estructural de virus, el ORF2 presenta las más altas tasas de variación de todos los aislados de PCV caracterizados hasta la fecha (Hamel y col., 1999). Por otra parte, el ORF1 codifica para una proteína esencial para la replicación viral $(\operatorname{Rep} P)$, la cual es transcrita en sentido inverso al ORF2 (Cheung, 2002). Al comparar las semejanzas de las secuencias de ORF1 de PCV-1 y PCV-2, se ha demostrado un $83 \%$ de homología entre sus nucleótidos y un $86 \%$ en las secuencias aminoacídicas predichas de sus proteínas. Por otra parte, se describe que la similitud en las secuencias de ORF2 entre PCV-1 y PCV-2 presenta un 67\% de homología para los nucleótidos y un $65 \%$ en la secuencias aminoacídicas predichas de sus proteínas (Mankertz y col., 2000). Las diferencias entre los genomas de PCV-1 y PCV-2 serían, aparentemente, la causa de la diferencia en virulencia entre PCV-1 y
PCV-2 (Hamel y col., 1998). Recientemente, un tercer gen viral llamado ORF3 ha sido descrito y caracterizado. Las proteínas de ORF3 parecen ser altamente conservadas entre cepas de $\mathrm{PCV}-2$ presentando más de un $95 \%$ de identidad a nivel de sus aminoácidos (Liu y col., 2005). Poco se conoce respecto a la significancia del resto de los ORFs.

\section{Distribución geográfica de PCV-2}

PCV-2 es considerado un virus ubicuo, presente en países en donde se han descrito o no enfermedades asociadas al circovirus porcino (PCVADs, del inglés, Porcine Circovirus Asociated Diseases) (Allan y Ellis, 2000).

Hasta la fecha, la infección por PCV-2 ha sido informada en Norteamérica, Europa (España, Francia, Reino Unido, Bélgica, Irlanda), Latinoamérica, Asia y África (Allan y Ellis, 2000). Estudios retrospectivos mostraron que el PCV-2 ha estado presente en Europa desde 1969 y en Canadá desde 1985. Las infecciones con PCV-2 han sido prevalentes en porcinos domésticos en todo el mundo desde algunas décadas y comúnmente el virus está de manera endémica presente en todos los países con industria porcina intensiva (Nauwynck y col., 2007).

\section{Transmisión de PCV-2}

Estudios experimentales, a la fecha, sugieren que el PCV-2 es de transmisión horizontal, por contacto directo, siendo considerada la vía oro-nasal la ruta más frecuente de infección entre animales infectados y susceptibles (Segalés y col., 2005). En granjas comerciales, la mayoría de los cerdos seroconvierten al PCV-2 entre los 2 y 4 meses de edad (Larochelle $y$ col., 2003), indicando que la transmisión horizontal del PCV-2 entre cerdos es muy eficiente y ha sido demostrada bajo condiciones experimentales en donde se pone en contacto a cerdos susceptibles con cerdos infectados (Albina y col., 2001; Bolin y col., 2001).

Las rutas de inoculación del PCV-2 tanto intranasal como subcutánea han sido usadas en intentos de reproducir experimentalmente el PMWS (Pogranichniy y col., 2000; Bolin y col., 2001).

La transmisión transplacentaria del PCV-2 ha sido recientemente demostrada mediante el seguimiento de infecciones experimentales intranasales en cerdos (Park y col., 2005), indicando que la transmisión vertical de PCV-2 es factible. Sin embargo, la frecuencia de estas alteraciones reproductivas bajo condiciones de campo es aparentemente variable, 
siendo raramente reportadas en Europa (Pensaert $y$ col., 2004; Maldonado y col., 2005), pero en Corea, en cambio, se han descrito infecciones por PCV-2 en aproximadamente un $13 \%$ de los fetos abortados y nacidos muertos (Kim y col., 2004).

Experimentalmente, se ha detectado excreción de PCV-2 en semen de verracos previamente inoculados con PCV-2. No obstante, se desconoce si las cantidades de PCV-2 presentes en semen pueden realmente producir la infección de las cerdas, ya sea por monta natural o por inseminación artificial (Larochelle y col., 2000), sin embargo, estas vías de infección deben ser consideradas como potenciales rutas de diseminación.

\section{Rutas de diseminación}

El PCV-2 puede ser detectado mediante la reacción en cadena de la polimerasa (PCR) en la cavidad nasal, tonsilas, secreciones bronquiales, heces y orina (Calsamiglia y col., 2004) tanto en cerdos afectados como libres de PMWS, pero la carga viral del virus en las secreciones es mucho mayor en cerdos afectados con PMWS (Calsamiglia y col., 2004). El virus puede ser aislado o detectado por PCR desde la cavidad nasal, rectal, vías urinarias, saliva, secreciones oculares y tonsilares (Krakowka y col., 2000; Bolin y col., 2001; Shibata $y$ col., 2003). Sin embargo, aunque se sabe que el PCV-2 puede ser detectado en la cavidad nasal y en la tonsila de cerdos cursando con PMWS por hibridación in situ (HIS), no se ha probado definitivamente si la carga viral obtenida por estas vías corresponde a excreción viral o si es debida a la presencia del virus en el ambiente, el cual alcanza esta localización (Segalés y col., 2004).

\section{Inmunidad frente a PCV-2}

Desde un punto de vista experimental, la seroconversión ocurre entre los 14 y 28 días postinfección (PI), no exhibiendo diferencias entre cerdos afectados subclínicamente con PCV-2 y cerdos cursando con PMWS (Krakowka y col., 2000; Pogranichniy y col., 2000; Resendes y col., 2001). Estudios de campo han demostrado que la seroconversión es usualmente detectada entre las 7 y 12 semanas de edad, como máximo hasta las 28 semanas. Aunque la respuesta humoral ocurra, un porcentaje variable de cerdos seropositivos puede permanecer virémico, sugiriendo que los anticuerpos detectados por IPMA o ELISA no son protectivos (Rodríguez-Arrioja y col., 2002).

Algunos estudios han demostrado cambios significativos en las subpoblaciones de células sanguí- neas periféricas como las células mononucleares de cerdos enfermos. En un estudio realizado en cerdos afectados con PMWS, se demostró un incremento de los monocitos circulantes, reducción de las células $\mathrm{T}$ (principalmente $\mathrm{CD}^{+}$) y de los linfocitos B y presencia de granulocitos inmaduros en baja densidad comparado con un cerdo clínicamente sano, no infectado por PCV-2 (Nielsen y col., 2001; Segalés y col., 2001). En conclusión, estos resultados sugieren una incapacidad de cerdos severamente infectados con PCV-2 para desarrollar una respuesta inmune efectiva.

\section{Células blanco de PCV-2}

Aunque hay certeza de que los síntomas clínicos se relacionan con desórdenes inmunopatológicos, los cerdos pueden ser asintomáticamente infectados. Algunos de los signos clínicos como la leucopenia sólo pueden evidenciarse en animales desarrollando PMWS, sin embargo, los animales que logran sobrevivir a la infección por PCV-2 desarrollan inmunidad contra el virus (McCullough y col., 2007).

La leucopenia asociada a cerdos desarrollando PMWS, es detectable antes del comienzo de los síntomas clínicos (Nielsen y col., 2003; Shibahara $y$ col., 2000). La leucopenia se manifiesta principalmente en los linfocitos B, seguido de los linfocitos $\mathrm{T}$, pero estos linfocitos (B y T) raramente portan el antígeno viral. Análisis in vivo han demostrado que el PCV-2 se asocia con mayor frecuencia a células monocíticas (monocitos, macrófagos y células dendríticas). Además de esta asociación persistente con las células dendríticas mielomonocíticas, los antígenos de PCV-2 pueden ser ocasionalmente encontrados en células endoteliales, células epiteliales y, como se mencionó anteriormente, en linfocitos (Darwich y col., 2004). Mientras que la infección in utero puede llevar a la acumulación de antígenos virales en los cardiomiocitos (Sánchez y col., 2003).

La asociación del virus con los linfocitos no se observa en todos los animales analizados y se manifiesta de manera transitoria, ya que el virus desaparece de los linfocitos a medida que el cerdo crece. Esta pérdida de la antígeno-positividad de los linfocitos se puede deber a la linfopenia resultado de la remoción de células positivas al virus. En contraste, todos los animales infectados por PCV-2, muestran antígenos virales en macrófagos y células dendríticas, los cuales aparentemente permanecen viables resultando una asociación persistente de estas células con el virus (Darwich y col., 2004). Análisis in vitro han confirmado la predilección del 
PCV-2 por las células dendríticas mielomonocíticas. Es más, estas células pueden albergar al antígeno viral por prolongados periodos, mientras que los linfocitos en un mismo cultivo permanecen sin infección. Además, el virus no aparenta replicar en las células dendríticas (Vincent y col., 2003; Gilpin $y$ col., 2003). Entonces surge la pregunta de dónde debería replicarse el virus para producir suficiente antígeno para mantener la alta carga viral encontrada en los macrófagos y células dendríticas. La respuesta se encuentra en estudios realizados in vitro en los cuales el PCV-2 va a infectar y replicarse en células del endotelio y epitelio intestinal de los cerdos, particularmente, cuando éstas células están cursando con algún tipo de respuesta inflamatoria local (Steiner $y$ col., 2007). La influencia de señales secundarias como la presencia de coinfecciones, inmunoestimulación o inmunosupresión resultan esenciales para afectar la inmunocompetitividad de los linfocitos ante una infección por PCV-2 (Allan y col., 2004; Segalés y col., 2005; Ellis y col., 2004; Ellis $y$ col., 1999).

\section{La inhibición de células dendríticas plasmocitoides por PCV-2 es la clave}

Las células dendríticas (CD) son las células presentadoras de antígeno más potentes del sistema inmune y tienen un rol muy importante en la inmunidad protectiva contra infecciones virales (Banchereau y Steinman, 1998). Como se señaló anteriormente, el PCV-2 es un virus que tiene tropismo por las células dendríticas, sin embargo no induce la muerte celular de éstas ni la de los linfocitos cocultivados con células dendríticas infectadas, por lo que no existe evidencia de transmisión del virus desde las CD infectadas a los linfocitos, incluso cuando éstos están activados (Vincent y col., 2003). Si bien PCV-2 no replica en células dendríticas, macrófagos ni monocitos (Vincent $y$ col., 2003, Gilpin y col., 2003), su sola presencia en estas células provoca cambios significativos en subpoblaciones celulares mononucleares de sangre periférica, llevando a una linfopenia, monocitosis y reducción de linfocitos B y T (Vincent y col., 2003, Gilpin y col., 2003, Nielsen y col., 2003; Shibahara $y$ col., 2000). La ausencia de evidencia de que PCV2 pueda infectar linfocitos, en conjunto con otros datos ha llevado a concluir que la depleción linfoide es producida por una vía indirecta, supuestamente por una desregulación (inhibitoria) de las señales inmunomoduladoras a nivel de células dentríticas plasmocitoides productoras de IFN- $\alpha$, generando una inmunosupresión severa por desregulación de la conexión existente entre el sistema inmune innato y adquirido del hospedero (Vincent $y$ col., 2007).

De esta forma PCV-2 además, se asocia con otros síndromes que afectan al cerdo, incluyendo el Complejo Respiratorio Porcino (CRP), la Neumonía Proliferativa Necrotizante, problemas reproductivos en cerdas gestantes, Síndrome de Dermatitis y Nefropatía Porcina (PDNS), en conjunto con otros agentes víricos como Parvovirus, PRRSV (Porcine Reproductive and Respiratory Syndrome virus), influenza, virus de la enfermedad de Aujeszky y agentes bacterianos como Mycoplasma pneumoniae, Actinobacillus pleuropneumoniae, Streptococcus suis y Pastereulla multocida (Allan y col., 2004, 1999; Krakowka y col., 2000, Rosell y col., 2000; West y col., 1999; Ohlinger y col., 2000). Actualmente todas estas enfermedades se conocen como PCVAD o enfermedades asociadas a circovirus porcino, nombre que la Asociación Americana de Veterinarios de Porcino (AASV) recomendó en marzo de 2006 para agrupar a todas las enfermedades atribuidas al PCV-2, incluida el PMWS.

\section{Caracterización genotípica de PCV-2}

El PCV-2 puede ser categorizado en diferentes genotipos según el análisis del polimorfismo de la longitud de los fragmentos de restricción (RFLP, del inglés restriction fragment length polymorphism) de su genoma, siendo una herramienta útil para la caracterización de los distintos aislados de PCV (Hamel y col., 1999). Estudios previos indican que las variaciones genéticas del PCV-2 deberían estar asociadas al origen geográfico más que con el tropismo celular o virulencia (Wen y col., 2005).

Desde la primera secuencia de PCV-2 descrita por Hamel y col. (1999), hasta julio del 2008, se han publicado aproximadamente 60 genotipos distintos de PCV-2 en GenBank, los cuales han sido aislados en distintos países y continentes (http://www.ncbi. nlm.nih.gov).

Recientemente, en China se realizó un estudio en donde se analizaron muestras de tejido de cerdos de distintas granjas, la presencia de PCV-2 fue detectada por PCR en un 59,9\% de las muestras analizadas (de diferentes regiones geográficas del país); éstas se sometieron, posteriormente, a análisis RFLP, distinguiéndose 9 patrones genéticos distintos del marco de lectura ORF2. El análisis filogenético de estos genotipos muestra que el PCV-2 de mayor prevalencia en China comprende tres grupos diferentes (Wen $y$ col., 2005). Un estudio de similares características fue realizado ante un brote de PMWS en Croacia en 
cuatro pequeños criaderos de cerdos, reportándose 4 secuencias del genoma de PCV-2 originadas de los aislados de campo (2099-M, 2099-1, 2099-2 y 2099-3). La región que codifica para la cápside (ORF2) fue secuenciada, y el análisis filogenético de esas cuatro secuencias los agrupó en un origen común, el cual está estrechamente relacionado con los genotipos de los aislados en Nueva Zelandia, Francia, China, UK y Eslovenia publicadas en GenBank (Jemersic y col., 2004).

Por otra parte, en Hungría, los genomas de PCV-2 aislados desde cerdos salvajes fueron divididos en 7 grupos (WB-H1-7), tres de los cuales presentaban un genoma de $1.767 \mathrm{bp}$ y los cuatro restantes $1.768 \mathrm{bp}$ de longitud. El análisis filogenético de las secuencias originó distintos tipos de árboles filogenéticos dependiendo de la fuente usada para la comparación. Interesantemente, el segmento rep (ORF1) se mantiene invariable entre los distintos grupos, mientras que al comparar el segmento cap (ORF2), nuevamente se revelan diferencias entre los grupos, sugiriendo la posibilidad de recombinación genómica entre distintos genotipos de PCV-2 (Cságola y col., 2005).

\section{En Chile existen subgenotipos de PCV-2}

El circovirus porcino tipo 2 (PCV-2) ha sido detectado en cerdos provenientes de 3 planteles de producción intensiva, ubicados en la zona central de Chile (Noriega 2008, Memoria Universidad de Chile). En este estudio se pudo detectar PCV-2, por medio de PCR, en el $100 \%$ de las muestras analizadas. Algunos de los genomas fueron secuenciados en el gen ORF2 para realizar la caracterización genotípica del virus en Chile. Estas secuencias se encuentran disponibles en Genbank bajo los siguientes números de acceso: EU186062, EU750909, EU519224, EU519223 http://www.ncbi.nlm.nih.gov/.

Un reciente estudio ha dividido el genoma de PCV-2 en 2 subgrupos o subgenotipos (Olvera $y$ col., 2007; Cheung y col., 2007). Esta clasificación se basa en las secuencias de ORF2 a nivel de los nucleótidos 262-267 y los correspondientes aminoácidos 88-89. El grupo 1 (genotipo PCV-2, subgenotipo 1) posee la secuencia nucleotídica CCCCGC en esta posición, la cual codifica para los aminoácidos prolina $\mathrm{y}$ arginina. Por otro lado, algunos representantes del grupo 1 además incluyen la secuencia CCCCTC, la cual codifica para los aminoácidos prolina y leucina. De esta manera, de acuerdo a esta clasificación, pertenecen al grupo 1 los subgenotipos que porten la secuencia CCCCG/TC, es esta posición. Por otra parte, el grupo 2 (genotipo PCV-2, subgenotipo 2), porta la secuencia de nucleótidos AAAATC, que codifica para lisina e isoleusina, es esta posición. Las últimas evidencias señalan que cepas originarias de Nueva Zelandia, Tailandia y el Reino Unido poseen sólo la secuencia nucleotídica CCCCG/TC (subgenotipo 1). Mientras que cepas de Japón, Canadá, España, Taiwán y Sudáfrica poseen sólo la secuencia AAAATC (subgenotipo 2). En contraste, cepas provenientes de Corea, Francia, Hungría, Austria, Alemania, Brasil y Estados Unidos comparten los 2 tipos de secuencias nucleotídicas (CCCCG/TC y AAAATC) (Dong-jun et al., 2007). Los genomas de ORF2 aislados en Chile tienen un rango promedio de $702 \mathrm{pb}$, y de acuerdo a las secuencias encontradas entre los nucleótidos 262-267, el genotipo nacional se clasificaría dentro del subgenotipo 1 (Noriega 2008).

Los análisis filogenéticos de todas las secuencias de PCV-2 reportadas en GenBank, reafirman, nuevamente, que la secuencia genética de ORF2 es la más apropiada para separar los aislados del virus en subgenotipos, pero hasta la fecha no existe una asociación clara de subgenotipos específicos con enfermedad o área geográfica (Olvera y col., 2007). Se ha especulado que las diferentes presentaciones clínicas que acompañan a la infección por el virus se deberían a variaciones genéticas entre los aislados de PCV-2 que se manifestarían con distintos grados de virulencia (Opriessnig y col., 2006). Es más, el estudio realizado por Opriessnig y col. (2006) sugiere que pequeñas diferencias en el genotipo de PCV-2 podrían manifestarse en considerables diferencias en su virulencia. Un análisis filogenético de ORF2 realizado en Suecia utilizando secuencias de granjas libres y afectadas con PMWS, demostró la existencia de 3 subgenotipos de PCV-2 (SG1-3), de acuerdo a un polimorfismo localizado en la posición 86-91 de la secuencia aminoácidica de la proteína Cap. Según este estudio, sólo el subgenotipo SG3 fue encontrado en cerdos enfermos (Timmusk y col., 2008). Anteriormente, la secuencia aminoacídica de ORF2 en esta misma posición (86-91) había sido propuesta por Cheung $y$ col. (Cheung y col., 2007) como un marcador genético para poder discriminar entre sungenotipos "viejos" y "nuevos" de PCV-2 presentes en Estados Unidos. Los resultados del estudio de Timmusk y col. 2008 reafirmarían de alguna manera esta hipótesis, ya que el marcador se corresponde con una de las regiones que facilita la diferenciación de los subgenotipos de PCV-2 pertenecientes a SG3, de los pertenecientes a SG2 o SG1. Dentro de la diferenciación única de aminoácidos para SG3, 8 se localizan entre las posiciones 57-91. 
En específico, en lugares 88 y 89 se reemplaza el aminoácido lisina $(\mathrm{K})$ e isoleucina (I), presentes en SG1 y SG2, por prolina (P) y arginina (R) presente exclusivamente en SG3, reflejando así un cambio de nucleótidos de AAA ATC a CCC CGC, constituyendo una característica única y representativa de SG3 (Timmusk y col., 2008). Los aislados chilenos presentan una relación con el subgrupo Sueco SG3, ya que a nivel del aminoácido 88 y 89 poseen prolina (P) y arginina (R) (Noriega 2008).

Ha sido sugerido que las sustituciones aminoácidicas en estas regiones han sido seleccionadas bajo la influencia de la respuesta inmune humoral del hospedero, sin embargo, aún no ha sido determinado si estas posiciones son importantes para el desarrollo de anticuerpos neutralizantes. Existe una correlación clara entre la carencia de anticuerpos neutralizantes contra PCV-2 y el desarrollo de PMWS, la cual ha sido demostrada por comparación de los niveles de anticuerpos en cerdos subclínicamente infectados y los que han desarrollado PMWS (Meerts y col., 2006). La importancia de la respuesta de anticuerpos contra PCV-2 en la protección contra PMWS es además respaldada por el valor protectivo que poseen los anticuerpos maternos contra PCV-2 (McKeown y col., 2005).

Existe evidencia creciente para la asociación de subgenotipos específicos con diferentes características de crecimiento in vitro, patogenicidad in vivo $\mathrm{y}$, tal vez lo más importante, diferencias epizoóticas de PMWS en el campo (Wen y col., 2005; Cheung y col., 2007; Chae, 2005). Los crecientes volúmenes de información de secuenciaciones disponibles y el concurrido uso de diferentes designaciones para la clasificación genotípica, vislumbran el requerimiento de acordar una nomenclatura internacional de genogrupos de PCV-2, la cual facilitaría enormemente la comparación entre los laboratorios de todo el mundo.

\section{Prevención y control}

Debido a que la infección por PCV-2 es ubicua y no se describen tratamientos específicos contra este síndrome, la prevención juega un rol esencial para controlar la infección. Entre las PCVDs, el PMWS es la enfermedad con el mayor impacto económico en producción porcina. Como definimos anteriormente, ésta es una enfermedad multifactorial en la que es necesaria la infección de los cerdos con PCV-2, pero, además, la influencia de factores infecciosos y no infecciosos que actúen de gatillos para el desarrollo de la forma clínica del síndrome. Por consiguiente, las medidas de control efectivas contra el PMWS se han centrado principalmente en el entendimiento de los cofactores que afectan a cada granja en particular y en el control y erradicación de éstos (Segalés $y$ col., 2005).

Estudios llevados a cabo en Francia desde 1998 han demostrado que las condiciones ambientales son necesarias, en asociación con PCV-2, para la expresión clínica de la enfermedad (Madec y col., 2000). A su vez, los circovirus porcinos son altamente resistentes a la inactivación con detergentes y desinfectantes comunes, haciendo difícil la descontaminación de los predios infectados, si es que no imposible (Allan y Ellis, 2000). Es interesante destacar que el primer brote de PCV-2 relacionado a PMWS descrito en Canadá ocurrió en un predio de cerdos de un estatus SPF y con altas medidas de bioseguridad (Ellis $y$ col., 1998; Harding, 1996; Harding y col., 1998). La implementación de lo que hoy en día es conocido como el plan de 20 pasos de Madec (lista de medidas de manejo que buscan disminuir el impacto de la enfermedad) ha disminuido significativamente el porcentaje de mortalidad en granjas severamente afectadas (Madec y col., 2001). Estas medidas fueron designadas para reducir la presión de infección en relación al PCV-2 y otras infecciones, a través de medidas de higiene y de reducción del estrés en las diferentes etapas de producción (Madec y col., 2000; Madec y Waddilove, 2002).

En los últimos años se han elaborado distintas vacunas que ayudan a la prevención de la infección por PCV-2. Actualmente, en EE.UU., Canadá y Europa se están ensayando 4 vacunas (Ingelvac de Boehringer; Suvaxyn de Fort Dodge; Circovac de Merial y una de Intervet, sin nombre). Estas están basadas en quimeras de versiones atenuadas del virus completo o subunidades proteicas recombinantes que involucran tanto a la proteína de la cápside (ORF2) como a la proteína que participa en la replicación viral (ORF1). En todos los casos se ha logrado la protección de lotes de cerdos vacunados aunque los resultados no son totalmente concluyentes. En Europa, por ejemplo, la vacuna más usada actualmente es Circovac (virus atenuado), disponible bajo licencia provisional y está empezando a exportarse al Reino Unido bajo un certificado de importación especial. En EE.UU. se está usando Ingelvac, la cual ha probado disminuir la mortalidad de los cerdos por PCV-2 significativamente. En la mayoría de los casos se ha observado un aumento en la tasa de crecimiento y una disminución en la tasa de mortalidad debido a la estimulación del sistema inmune adaptativo y el traspaso de grandes niveles de anticuerpos maternos a los lechones (Burch, 2007). 
Aparentemente, una buena bioseguridad no nos asegura estar libre de las enfermedades asociadas al PCV-2. Un diagnóstico rápido, la pronta remoción de los animales enfermos de las granjas y buenas prácticas de manejo serían las únicas medidas para controlar las pérdidas atribuidas a la infección por PCV-2 (Allan y Ellis, 2000).

\section{REFERENCIAS}

Albina, E.; Truong, C.; Hutet, E.; Blanchard, P.; CARiolet, R.; L'Hospitalier, R., Mahe, D.; Allee, C., Morvan, H.; Amenna, N.; Ledimma, M.; MadeC, F.; Jestin, A. 2001. An experimental model for post-weaning multisystemic wasting syndrome (PMWS) in growing piglets. J. Comp. Pathol. 125:292-303.

Allan, G.; PheniX, K.; TodD, D.; McNulty, M. 1994. Some biological and physico-chemical properties of porcine circovirus. J. Vet. Med. B. 41:17-26.

Allan, G.; McNeilly, F, Meehan, B.; Kennedy, S.; Mackie, D.; Ellis, J.; Clark, E.; EsPuÑa, E.; SAubi, N.; Riera, P.; BOTNER, A. y CHARREYRE, C. 1999. Isolation and characterisation of circoviruses from pigs with wasting syndromes in Spain, Denmark and Northern Ireland. Vet. Microbiol. 66:115-123.

Allan, G.; MCNeIlly, F.; Ellis, J.; KraKowKa, S.; Botner, A.; MCCUllough, K.; NAUWYNCK, H.; KenNedy, S.; MeEhan, B.; Charreyre, C. 2004. PMWS: experimental model and co-infections. Vet. Microbiol. 98:165-168.

Allan, G; McNeilly, F.; KenNedy, S.; DAFt, B.; Clark, E.; ELLIS, J.; HAINES, D.; MEEHAN, B.; ADAIR, B. 1998. Isolation of porcine circovirus-like viruses from pigs with a wasting disease in the USA and Europe. J. Vet. Diagn. Invest. 10:3-10.

Allan, G. y Ellis, J. 2000. Porcine circoviruses: A review. J. Vet. Diagn. Invest. 12:3-14.

BAnchereau, J. y Steinman, R. 1998. Dendritic cells and the control of immunity. Nature. 392:245-252.

BASSAMI, M.; BerRyman, D.; WilcoX, G. y RAIDAL, S. 1998. Psittacine beak and feather disease virus nucleotide sequence analysis and its relationship to porcine circovirus, plant circoviruses, and chiken anaemia virus. Virology. 249:453-459.

Bolin, S.; Stoffregen, W.; Nayar, G.; Hamel, A. 2001. Postweaning multisystemic wasting syndrome induced after experimental inoculation of cesarean-derived, colostrums deprived piglets with type 2 porcine circovirus. J. Vet. Diagn. Invest. 13:185-194.

BURCH, D.; 2007. PCV-2 vaccines are on their way. [En línea]. $<$ http://www.octagon-services.co.uk/articles/PCV-2vaccines. htm> [consulta: 29- 07-2007].

Calsamiglia, M.; Olvera, A.; Segalés, J.; Domingo, M. 2004. Quantification of PCV-2 in different routes of excretion: possible transmission routes and correlation with presence of PMWS characteristic lesions. Proceedings of the International Pig Veterinary Society Congress. P. 11.

CHAE, C. 2005. A review of porcine circovirus 2-associated syndromes and diseases. Vet. J. 169:326-336.

CheUnG, A. K. 2002. Transcriptional Analysis of Porcine Circovirus Type 2. J. Virol. 305:168-180.
Cheung, A.; LAgER, K.; KohutyuK, O.; VinCent, A.; Henry, S.; BAKER, R.; Rowland, R.; DUNHAM, A. 2007. Detection of two porcine circovirus type 2 genotypic groups in United States swine herds. Arch. Virol. 152:1035-1044.

CLARK, E. 1997. Post-weaning multisystemic wasting syndrome. Proceedings of the American Association of Swine Practitioners. 28:499-501.

CSÁGOla, A.; KeCSKemÉti, S.; KISS, I.; Tuboly, T. 2005. Genetic characterization of type 2 porcine circoviruses detected in Hungarian wild boars. Arch. Virol. 151:495-507.

DARWich, L.; Segalés, J.; Mateu, E. 2004. Pathogenesis of postweaning multisystemic wasting syndrome caused by porcine circovirus 2: an immune riddle. Arch. Virol. 149:857-874.

DONG-JUN, A.; IN-SOON, R.; DAE-SUB, S.; CHOI-KYU, P.; BONGKYUN, P. 2007. Phylogenetic characterization of porcine circovirus type 2 in PMWS and PDNS Korean pigs between 1999 and 2006. Virus Res. 129:115-122.

Ellis, J.; Clark, E.; Haines, D.; West, K.; KraKowKa, S.; KeNNEDY, S.; ALLAN, G. 2004. Porcine circovirus-2 and concurrent infections in the field. Vet. Microbiol. 98:159-163.

Ellis, J.; Hassard, L.; Clark, E.; Harding, J.; Allan, G.; Willson, P.; Strokappe, J.; Martin, K.; MCNeilly, F.; MEEHAN, B.; TODD, D. y HAINES, D. 1998. Isolation of circovirus from lesions of pigs with postweaning multisystemic wasting syndrome. Can. Vet. J. 39:44-51.

Ellis, J.; Krakowka, S.; LaIRmore, M.; Haines, D.; BrataNICH, A.; ClarK, E.; Allan, G.; KonOby, C.; Hassard, L.; Meehan, B.; Martin, K.; Harding, J.; Kennedy, S.; MCNEILly, F. 1999. Reproduction of lesions of postweaning multisystemic wasting syndrome in gnotobiotic piglets. J. Vet. Diagn. Invest. 11:3-14.

Gilpin, D.; Mccullough, K.; Meehan, B.; Mcneilly, F.; MCNAIR, I.; STEVENSON, L.; FOSTER, J.; ElLIS, J.; KRAKOWKA, S.; ADAIR, B.; ALLAN, G. 2003. In vitro studies on the infection and replication of porcine circovirus type 2 antigen in cells of the porcine immune system. Vet. Immunol. Immunopathol. 94:149-161.

HAMEL, A.; Lin, L.; NAYAR, G. 1998. Nucleotide sequence of porcine circoviruses associated with postweaning multisystemic wasting syndrome in pigs. J. Virol. 72:5262-5267.

HAMel, A.; Lin, L.; SACHVIE, C.; GRUdeski, E.; NAYAR, G. 1999. PCR detection and characterization of type-2 porcine circovirus. Can. J. Vet. Res. 64:44-52.

HARDING, J. 1996. Post-weaning multisystemic wasting syndrome: preliminary epidemiology and clinical findings. Proceedings of the Western Canadian Association of Swine Practitioners. P. 21.

Harding, J; Clark, E.; Strokappe, J. 1998. Post-weaning multisystemic wasting syndrome (PMWS): epidemiology and clinical presentation. Swine Health Prod. 6:249-254.

HARDING, J. y ClARK, E. 1997. Recognizing and diagnosing postweaning multisystemic wasting syndrome (PMWS). Swine Health Prod. 5:201-203.

Jemersic, L.; Cvetnic, Z.; ToplaK, I.; Spicic, S.; Grom, S.; BarliC-MAganja, D.; Terzic, S.; HostnIK, P.; LOJKIC, M.; HUMSKI, A.; HABRUN, B.; KRT, B. 2004. Detection and genetic characterization of porcine circovirus type $2(\mathrm{PCV}-2)$ in pigs from Croatia. J. Vet. Sci. 77:171-175.

Jestin, A.; Mahe, D.; Blanchard, P y Boisseson, C. 2001. Porcine circoviruses. Proceedings of the ssDNA Viruses of plants, birds, pigs and primates: porcine post-weaning multisystemic wasting syndrome. European Society of Veterinary Virology. 32. 
KIM, J.; JunG, K.; CHAE, C. 2004. Prevalence of porcine circovirus type 2 in aborted fetuses and stillborn piglets. Vet. Rec. 155:489-492.

KrakowKa, S.; Ellis, J.; MeEhan, B.; KenNedy, S.; MCNeIlly, F.; ALLAN, G. 2000. Viral wasting syndrome of swine: experimental reproduction of postweaning multisystemic wasting syndrome in gnotobiotic swine by coinfection with porcine circovirus 2 and porcine parvovirus. Vet. Path. 37:254-263.

Larochelle, R.; Bielanski, A.; Muller, P.; Magar, R. 2000. PCR detection and evidence of shedding of porcine circovirus type 2 in boar semen. J. Clin. Microbiol. 38:4629-4632.

LAROCHELle, R.; MAGAR, R.; D’AlLAIRE, S. 2002. Genetic characterization and phylogenetic analysis of porcine circovirus type 2 (PCV-2) strains from cases presenting various clinical conditions. Virus Res. 90:101-112.

LAROChelle, R.; Magar, R.; D'Allaire, S. 2003. Comparative serologic and virologic study of commercial swine herds with and without postweaning multisystemic wasting syndrome. Can. J. Vet. Res. 67:114-120.

LiU, J.; ChEN, I.; KWANG, J. 2005. Characterization of a previously unidentified viral protein in porcine circovirus type 2 infected cells and its role in virus induced apoptosis. J. Virol. 79:8262-8274.

Madec, F.; Eveno, E.; Morvan, P.; Hamon, L.; Blanchard, P.; Cariolet, R.; Amenna, N.; Morvan, H.; Truong, C.; Mahe, D.; Albina, E. y Jestin, A. 2000. Post-weaning multisystemic wasting syndrome (PMWS) in pigs in France: clinical observations from follow-up studies on affected farms. Livest. Prod. Sci. 63:223-233.

Madec, F.; Rose, N.; Eveno, E.; Morvan, P.; Larour, G.; Jolly, J.; Le Diguerher, G.; CARIOLET, R.; Le Dimna, M.; BlaNCHARD, P. y JESTIN, A. 2001. PMWS: on-farm observations and preliminary analytic epidemiology. In: Proceedings of the ssDNA Viruses Plants, Birds, Pigs and Primates (ESVV) Meeting. Pp. 86-87.

MADEC, F. y WADDILOVE, J. 2002. Control PCV-2 or control other factors? Several approaches to a complex problem. In: PMWS and PCV-2 Diseases: Beyond the Debate. Merial Symposium, Ames, IA, USA. Pp. 45-53.

Mccullough, C.; Vincent, E.; SUmmerfield, A.; KrakowKA, S.; Ellis, A.; SegalÉs, J.; Allan, G.; 2007. The immunology of PCV-2 infections. American Assoc. of Swine Vet. Pp. 497-500.

MCKeOWn, N.; OpriesSniG, T.; ThOMAS, P.; GuenetTe, F.; ELVINGER, M.; FENAUX, M.; HALBUR, P.; MENG, X. 2005. Effects of porcine circovirus type $2(\mathrm{PCV}-2)$ maternal antibodies on experimental infection of piglets with PCV-2. Clin. Diagn. Lab. Immunol. 12:1347-1351.

Maldonado, J.; Segalés, J.; MartineZ-PUig, D.; CAlsamiglia, M.; RIERA, P; DOMINGO, M.; ARTIGAS, C. 2005. Identification of viral pathogens in aborted fetuses and stillborn piglets from cases of swine reproductive failure in Spain. Vet. J. 169:454-456.

MANKeRTZ, A.; DOMINDO, M.; Folch, J.; LeCANN, P.; Jestin, A.; SEgalés, J.; ChMielewicz, B.; Plana-durÁn, J.; SoIKe, D. 2000. Characterisation of PCV-2 isolates from Spain, Germany and France. Virus Res. 66:65-77.

Meehan, B.; Creelan, J.; McNulty, M.; Todd, D. 1997. Sequence of porcine circovirus DNA: affinities with plant circoviruses. J. Gen. Virol. 78:221-227.

Meehan, B.; MCNeilly, F.; TodD, D.; Kennedy, S.; JeWhURST, V., Ellis, J.; Hassard, L., Clark, E., Haines, D.; Allan,
G. 1998. Characterization of novel circovirus DNAs associated withwasting syndromes in pigs. J. Gen. Virol. 79:2171-2179.

MeERTS, P.; Misinzo, G.; LefebVRe, D.; Nielsen, J.; Botner, A.; KRISTENSEN, C.; NAUWYNCK, H. 2006. Correlation between the presence of neutralizing antibodies against porcine circovirus type 2 (PCV-2) and protection against replication of the virus and development of PCV-2 associated diseases. BMC Vet. Res. 2:1-6.

NaUwynck, H.; LefebVre, D.; Misinzo, G.; MeERTs, P.; MAteusen, B.; Sanchez, R.; Delputte, P. 2007. Pathogenesis of porcine circovirus 2 infections. American Assoc. of Swine Vet. pp. 489-493.

Niegro, F.; Forsthoefel, A. y LAWTHer, R. 1998. Beak and feather disease virus and porcine circovirus genomes; intermediates between the geminiviruses circoviruses. Arch Virol. 143:1723-1744.

NiElSEN, J.; Vincent, E.; LAdEKJAER-MikKelsen, A.; Allan, G.; MCCULLOUGH, K.; BOTNER, A. 2001. Experimental infection of 3-week-old piglets with PCV-2 altered the level of various peripheral blood leukocyte population. Proceedings of the ssDNA Viruses of Plants, Birds, Pigs and Primates: porcine post-weaning multisystemic wasting syndrome. European Society of Veterinary Virology. P. 96.

NiELSEN, J.; VINCENT, E.; BOTNER, A.; LADEKJAER-MIKKELSEN, A.; Allan, G.; Summerfield, A.; MCCullough, C. 2003. Association of lymphopenia with porcine circovirus type 2 induced postweaning multisystemic wasting syndrome (PMWS). Vet. Immunol. Immunopathol. 92:97-111.

NORIEGA JORGE. 2008. Detección y Caracterización Genotípica de Circovirus Porcino tipo 2 PCV-2 en Chile. Memoria para optar al título de Médico Veterinario. Facultad de Cs. Veterinarias y Pecuarias. Universidad de Chile.

OHLINGER, V.; SCHMIDT, U.; PESCH, S. 2000 Studies on pathogenic aspects of the post-weaning multisystemic wasting syndrome (PMWS). Proceedings of the $16^{\text {th }}$ International Pig Veterinary Society Congress.

Olvera, A.; CORTEY, M.; Segales, J. 2007. Molecular evolution of porcine circovirus type 2 genomes: Phylogeny and clonality. Virology. 357:175-185.

Opriessnig, T.; MCKown, N.; Zhon, E.; Mong, X.; Halbur, P. y GEN, J. 2006. Genetic and experimental comparison of porcine circovirus type $2(\mathrm{PCV}-2)$ isolates from cases with and without PCV-2-associated lesions provides evidence for differences in virulence. J. Gen. Virol. 87:2923-2932.

PARK, J.; KIM, J.; HA, Y.; JunG, K.; CHOI, C.; LiM, J.; KIM, S.; CHAE, C. 2005. Birth abnormalities in pregnant sows infected intranasally with porcine circovirus 2 . J. Comp. Pathol. 132:139-144.

Pensaert, M.; SAnchez, JR R.; LadekJaer-MikKelsen, A.; ALLAN, G.; NAUWYNCK, H. 2004. Viremia and effect of fetal infection with porcine viruses with special reference to porcine circovirus 2 infection. Vet. Microbiol. 98:175-183.

Phenix, K.; Weston, J.; Ypelaar, I.; Lavazza, A.; Smith, J.; TodD, D.; Wilcox, G. y RaIdAL, S. 2001. Nucleotide sequence analysis of a novel circovirus of canaries and its relationship to other members of the genus circovirus of the family circoviridae. J. Gen. Virol. 82:2805-2809.

Pogranichniy, R.; Yoon, K.; Harms, P.; Swenson, S.; ZimMERMAN, J.; SORDEN, S. 2000. Characterization of immune response of young pigs to porcine circovirus type 2 infection. Viral Immunol. 13:143-153. 
Resendes, A.; Balasch, M.; Calsamiglia, M.; Segalés, J.; Sibila, M.; Mankertz, A.; Plana-Durán, J.; Domingo, M. 2001. Experimental co-inoculation of porcine circovirus type 2 (PCV-2) and a vaccine adjuvant in conventional pigs. Proceedings of the ssDNA Viruses of Plants, Birds, Pigs and Primates: porcine post-weaning multisystemic wasting syndrome. European Society of Veterinary Virology. P. 134.

Rodríguez-Arrioja, G.; Segalés, J.; Calsamiglia, M.; Resendes, A.; Balasch, M.; Plana-Durán, J.; CASAl, J.; DomINGO, M. 2002. Dynamics of porcine circovirus type 2 infection in a herd of pigs with postweaning multisystemic wasting syndrome. Am. J. Vet. Res. 63:354-357.

Rosell, C.; Segalés, J.; Plana-DuRÁn, J.; BAlasch, M.; RoDRÍGUEZ-ARRIOJA, G.; KENNEDY, S.; ALlAN, G.; MCNEILly, F.; LATIMER, K. y DOMINGO, M. 1999. Pathological, inmunohistochemical, and in-situ hybridization studies of natural cases of postweaning multisystemic wasting syndrome (PMWS) in pigs. J. Comp. Path. 120:59-78.

Rosell, C., Segalés, J.; Ramos-VARA, J.; Folch, J.; RodrIGUEZ-ARrioja, G.; DuRAn, C.; BALASCh, M.; PlanA-DurÁN, J.; DomingO, M. 2000. Identification of porcine circovirus in tissues of pigs with porcine dermatitis and nephropathy sindrome. Vet. Rec. 146:40-43.

SANCHEZ, R.; MEERTS, P.; NAUWYNCK, H.; PENSAERT, M. 2003. Change of porcine circovirus 2 target cells in pigs during development from fetal to early postnatal life. Vet. Microbiol. 95:15-25.

Segalés, J.; Alonso, F.; Rosell, C.; PAstor, J.; Chianini, F.; CAMPOS, E.; LÓPES-FuERTES, L.; QUINTANA, J.; RODRIGUEZArrioja, G.; CAlsamiglia, M.; Pujols, J.; DomíngueZ, J.; DomingO, M. 2001.Changes in peripheral blood leukocyte populations in pigs with natural postweaning multisystemic wasting syndrome(PMWS). Vet. Immunol. Immunopathol. 81:37-44.

Segalés, J. y Domingo, M. 2002. Postweaning multisystemic wasting syndrome (PMWS) in pigs. A review. Vet Q. 24:109-124.

Segalés, J.; Rosell, C.; Domingo, M. 2004. Pathological findings associated with naturally acquired porcine circovirus type 2 associated disease. Vet. Microbiol. 98:137-149.

Segalés, J.; Allan, G.; Domingo, M. 2005. Porcine circovirus diseases. Anim. Health Res. Rev. 6:119-142.

Steiner, E.; Balmelli, C.; Vincent, E.; Summerfield, A.; MCCullough, K. 2007. Multipotent cell targeting by PCV-2. J. Virol. (Submited for publication).
ShiBAhara, T.; SATO, K.; ISHAKaWA, Y.; KADOTA, K. 2000. Porcine circovirus induces $\mathrm{B}$ lymphocyte depletion in pigs with wasting disease syndrome. J. Vet. Med. Sci. 62:1125-1131.

Shibata, I.; OKUda, Y.; YaZaWA, S.; OnO, M.; SASAKI, T.; ITAGAKI, M.; NAKAJIMA, N.; OKABE, Y.; HideJiMA, I. 2003. PCR detection of porcine circovirus type 2 DNA in whole blood, serum, oropharyngeal swab, nasal swab, and feces from experimentally infected pigs and field cases. J. Vet. Med. Sci. 65:405-408.

TIMMUSK, S.; WALLGREN, P.; BRUNBORG, I.; WIKSTROM, F.; ALLAN, G.; MeEhan, B.; McMenamy, M.; MCNeIlly, F.; FuXler, L.; Belak, K.; Poderosoo, D.; SAAR, T.; BerG, M.; Fossum, C. 2008. Phylogenetic analysis of porcine circovirus type 2 (PCV2) pre- and post-epizootic postweaning multisystemic wasting syndrome (PMWS). Virus Genes. 36:509-520.

TISCHER, I; RASH, R; TOCHTERMANN, G. 1974. Characterization of papovavirus- and picornavirus- like particles in permanent pig kidney cell lines. Zentralbl. Bakteriol. Hyg A. 226:153-167.

Tischer, I.; Gelderblom, H.; VettermanN, W.; KoCH, M.A. 1982. A very small porcine virus with circular single-stranded DNA. Nature. 295:64-66.

TODD, D.; Creelan, J.; L., MACKIE, D.; RixOn, F. y McNulty, M. 1990. Purification and biochemical characterisation of chicken anaemia agent. J. Gen. Virol. 71:819-823.

TodD, D., Weston, J.; Solke, D. y Smyth, J. 2001. Genome sequence determinations and analyses of novel circoviruses from goose and pigeon. Virology. 286:354-362.

Vincent, E.; Carrasco, C.; Herrmann, B.; Meehan, M.; Allan, G.; Summerfield, A.; McCullough, K. 2003. Dendritic cells harbor infectious porcine circovirus type 2 in the absence of apparent cell modulation or replication of the virus. J. Virol. 77:13288-13300.

Vincent Ie, Balmelli C, MeEhan B, Allan G, Summerfield A, MCCullOugh KC. 2007. Silencing of natural interferon producing cell activation by porcine circovirus type 2 DNA. Immunology. 120:47-56.

Wen, L.; GuO, X.; YANG, H. 2005. Genotyping of porcine circovirus type 2 from a variety of clinical conditions in China. J. Vet. Microbiol. 110:141-146.

West, K.; BYSTROM, J.; WoJnAROWICZ, C.; SHANTZ, N.; JACOBson, M.; Allan, G.; Haines, E.; Clark, E.; KraKowKa, S.; McNeilly, F.; Konoby, C.; Martin, K.; Ellis, J. 1999. Myocarditis and abortion associated with intrauterine infection of sows with porcine circovirus 2. J. Vet. Diagn. Invest. 11:530-532. 